\title{
Modelagem sísmica no fluxo de trabalho exploratório
}

Daina Popic Bendoraitis ${ }^{1}$, Danilo de O. Domingos ${ }^{1}$, Felipe de Souza Duarte ${ }^{1}$, Mauren Paola Ruthner ${ }^{1}$, Gerson Luis da Silva Ritter $^{1}$ e Luiz Alberto Santos ${ }^{1,2}$.

1- Petrobras; 2-Universidade Federal Fluminense.

\section{Copyright 2021, SBGf - Sociedade Brasileira de Geofísica}

This paper was prepared for presentation during the $17^{\text {th }}$ International Congress of the Brazilian Geophysical Society held in Rio de Janeiro, Brazil, 16-19 August 2021.

Contents of this paper were reviewed by the Technical Committee of the $17^{\text {th }}$ International Congress of the Brazilian Geophysical Society and do not necessarily represent any position of the SBGf, its officers or members. Electronic reproduction or storage of any part of this paper for commercial purposes without the written consent of the Brazilian Geophysical Society is prohibited.

\section{Abstract}

Seismic modeling is an important tool for exploration, production development and production in oil industry. It is suitable for supporting acquisition design, for testing of processing algorithms and for evaluating scenarios for seismic interpretation. A workflow involving model building, seismic modeling and data evaluation is described and applied in a locus between Campos and Santos Basin. For model building we use a workflow called SISMEC-acustico to construct elastic models. The SISMEC model has enough details to perform seismic modeling with wave equation formulation using finitedifference numerical method. The model is submitted to wave equation with elastic formulation. Synthetic and real data are compared revealing excellent kinematic correspondence between main events which testifies an acceptable Vp to represent subsurface reality.

\section{Introdução}

A modelagem sísmica constitui poderosa ferramenta para o segmento de exploração e produção da indústria do petróleo. O aumento do poder computacional e a constante necessidade de redução de riscos e custos, naturalmente, causaram correspondente intensificação da modelagem sísmica para dar suporte à aquisição, ao processamento e à interpretação.

A simulação de propagação de ondas é um campo de pesquisa que teve início por volta dos anos 1970's com o desenvolvimento da tecnologia computacional e algoritmos para solução numérica de equações diferenciais (Carcione, 2002). Atualmente, a revista Geophysics lista mais de 40000 trabalhos versando sobre o tema modelagem sísmica.

Nos anos 1990 e, mais intensamente nos anos 2000 e seguintes, houve extraordinário número de publicações e teses produzidos com foco em modelagem, entre eles destacam-se: Cunha (1992) abordando a modelagem elástica; Rosa Filho (2002) descrevendo e implementando a modelagem elástica com anisotropia; Martins (2003) implementando a modelagem elástica anisotrópica com a formulação velocidade-tensão; Bulcão (2004) que desenvolveu uma modelagem e migração acústica e elástica; Lira (2004) que simulou os efeitos do acamamento delgado na propagação com algoritmo acústico, Alves et al. (2008) que realizaram estudos de iluminação com algoritmo acústico e Duarte (2012) que desenvolveu uma modelagem acústica na frequência.

Este trabalho tem como objetivo empregar a modelagem para testar um cenário para dar suporte à interpretação e avaliar o campo de velocidade obtido durante o processamento sísmico. Neste sentido, à luz do conhecimento adquirido seja na representação de modelos, seja na simulação numérica da Ondulatória, a incorporação da modelagem no fluxo de trabalho da cadeia sísmica auxilia a melhor compreensão da subsuperfície mitigando riscos exploratórios.

Um fluxo de trabalho envolvendo modelagem contempla 4 etapas, a saber: (1) Construção de modelo, dedicada a criação de fluxos e metodologias para construção de modelos de velocidade $P$, velocidade $S$, densidade $e$ demais propriedades úteis na simulação da ondulatória com formulação elástica; (2) Modelagem, que se dedica a avaliação das rotinas de modelagem sísmica, criação de fluxos de modelagem, parametrizações adequadas a cada problema e formas de apresentação dos resultados; (3) Processamento, fase na qual os dados gerados serão processados e subsidiará a criação do fluxo de trabalho com proposição de cenários para refazimento da modelagem; (4) Análise, fase na qual serão consolidados os fluxos de forma integrada e proposição de formas de avaliação dos resultados.

Este trabalho apresenta os resultados da etapa 1 e os primeiros ensaios da etapa 2 envolvendo um setor entre as Bacias de Santos e Campos.

\section{Metodologia}

A criação de modelos guiados pela sísmica, permite gerar volumes de propriedades elásticas sem necessidade direta de poços e horizontes. Neste trabalho exibimos os resultados de aplicação das etapas iniciais do fluxo SISMEC-Acústico (da SíSmica à geoMECânica) (Santos et al., 2017) para criação de modelos mecânicos compostos por volumes de velocidade $\mathrm{P}\left(v_{p}\right)$, velocidade

S $\left(v_{s}\right)$ e densidade $(\rho)$ empregando-se um fluxo de trabalho derivado de inversão acústica.

O fluxo SISMEC-Acústico foi aplicado a um dado adquirido nas Bacias de Santos e Campos, reprocessado recentemente, que possui uma área de aproximadamente $20 \times 40 \mathrm{~km}$.

O SISMEC empregado neste projeto tem início com o 
método de inversão acústica cujos insumos são o volume sísmico de amplitudes processado e o campo de velocidade de migração correspondente. Sempre que disponíveis, horizontes e perfis de poços são úteis para estimativa de propriedades e de relações entre parâmetros, bem como para o cálculo dos volumes de propriedades e o controle de qualidade.

A ideia central é combinar um volume de impedância com maior conteúdo de baixas frequências, proveniente da velocidade de processamento, com outro rico em médias e altas frequências, com origem na sísmica (Figura 1). A partir deste volume de impedância combinado são recalculadas as propriedades $v_{p}{ }^{\prime}, v_{s}{ }^{\prime}$ e $\rho$, que deverão apresentar maior riqueza de detalhes.

A seguir são descritas as etapas para obtenção destes volumes, ilustradas no fluxograma da Figura 2.

1. Conversão para tempo: Nesta etapa o volume da sísmica é convertido para tempo através de raio vertical.

2. Deconvolução iterativa: Nesta etapa a sísmica tem o pulso residual deconvolvido, aumentando seu conteúdo de frequências.

3. Pseudo Impedância de Alta Resolução (médias e altas frequências): Nesta etapa 0 dado deconvolvido é integrado, com tratamento especial das baixas frequências com suavização por estruturas, para a obtenção da pseudo-impedância. Neste momento é importante que o produto seja zerado do fundo do mar para cima.

4. Conversão para profundidade: A conversão para profundidade é realizada por raio vertical da mesma forma que a conversão para tempo, apenas alterando o sentido. Importante notar que o fluxo até esta etapa é um procedimento convencional e amplamente aplicado no processamento de dados sísmicos.

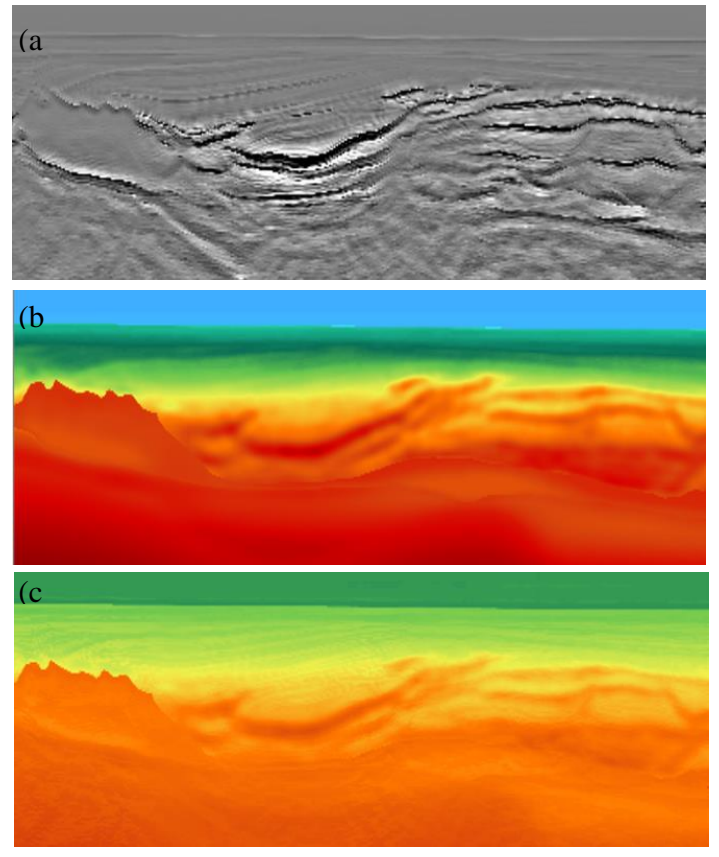

Figura 1. (a) Pseudo-impedância derivada da sísmica; (b) Impedância derivada da velocidade; (c) Volume de impedância resultado da combinação de (a) e (b) por meio da ferramenta "Combina Volumes", disponível no WebSintesi.

5. Cálculo do modelo de baixas frequências: A impedância proveniente da velocidade é calculada por meio da expressão:

$I=V_{p} . \rho$

onde $v_{p}$ corresponde à velocidade $P$ e $\rho$ representa a densidade. $O$ volume de densidades, por sua vez, pode ser calculado por ajuste da equação de Gardner, conforme a expressão abaixo:

$$
\rho=a v_{p}^{b}
$$

sendo que $a$ e b são parâmetros do ajuste do tipo potencial, obtidos com dados de poços. Para a região da água, impõe-se que a densidade seja igual à densidade do citado fluido. Cabe ressaltar que tipos litológicos diferentes apresentam relações diferentes e nem sempre todas as litologias são perfiladas, sendo necessário um controle de qualidade para averiguar a validade destas relações

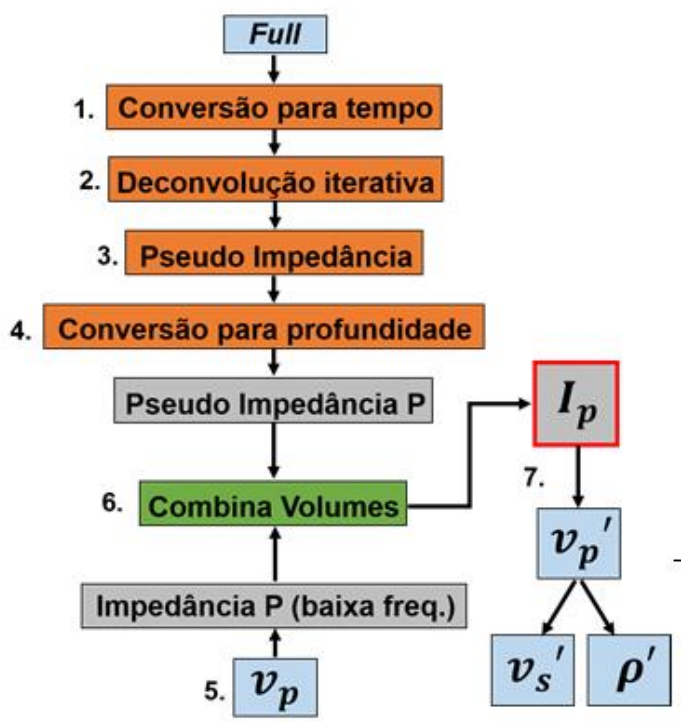

Figura 2. Fluxo empregando inversão acústica.

6. Ajuste entre os modelos de baixa, média e altas frequências: Para esta etapa é utilizada a ferramenta "Combina Volumes", disponível no WebSintesi (sistema de processamento interpretativo da Petrobras).

7. Recálculo de propriedades: Na posse da impedância $P$ é possível recalcular as propriedades básicas: $v_{p}{ }^{\prime}, \rho^{\prime} \mathrm{e}$ $v_{s}^{z}$, que contam com todo o detalhamento introduzido pelo fluxo em questão. Para tal, é realizado o caminho inverso do fluxo empregando as relações de Gardner já 
citadas na equação (2).

$$
\begin{aligned}
& v_{p}^{I}=\left(\frac{I_{p}}{a}\right)^{\frac{1}{b+1}} \\
& \rho^{\prime}=a v_{p}^{\prime b} \ldots .
\end{aligned}
$$

A velocidade $\mathrm{S}, v_{s}^{I}$, pode ser calculada por ajuste da equação de Castagna:

$$
v_{s}^{\prime}=m v_{p}{ }^{\prime}-c
$$

onde $m$ e $c$ são parâmetros obtidos com dados de poços.

\section{Resultados}

Os tópicos a seguir apresentam os resultados da etapa 1 do projeto, geração do modelo, e os primeiros ensaios da etapa 2, modelagem. Os resultados são apresentados para a linha A (2D), Figura 6 superior, da região de estudo.

\section{Modelo V0}

Empregando o fluxo de trabalho descrito no tópico anterior foi gerada a primeira versão do modelo $3 \mathrm{D}$

contemplando as propriedades $v_{p}, v_{s}$ e densidade.

As Figuras 3, 4 e 5 exibem estas propriedades para a linha A do volume sísmico em estudo. São feitas comparações antes e após a aplicação da ferramenta "Combina Volumes".

A Figura 3 superior constitui a velocidade obtida com processamento Full Waveform Inversion - FWI (cortesia da CGG). Este campo exibe estruturação complexa e contrastes fortes de velocidade, seja pelo sal ou por intrusões e derrames ladeados por unidades sedimentares. A Figura 3 inferior exibe 0 resultado da aplicação da ferramenta mencionada. Ela mostra o substancial aumento da riqueza de detalhes e se apresenta bastante condizente com a geologia esperada.

Da mesma forma, as Figuras 4 e 5 mostram o aumento da riqueza de detalhes e de contrastes entre camadas

nas propriedades $v_{s} \mathrm{e} \rho$, respectivamente.

\section{Modelagem}

A etapa de modelagem foi conduzida com um algoritmo de propagação elástica e os insumos fornecidos pela metodologia SISMEC-Acústico. Os resultados alcançados até 0 momento apresentam ótima compatibilidade cinemática com o dado real. Tomando como exemplo a linha mencionada ao longo do trabalho, verificam-se que os principais refletores imageados no dado real, na Figura 6 superior, estão de acordo com a imagem migrada utilizando o dado sísmico modelado (Figura 6 inferior). Considerando que abordagem cinemática foi adequada, o próximo passo a se tratar é a compatibilização das amplitudes, que encerram diferenças significativas entre dado real e sintético.

\section{Conclusões}

A metodologia SISMEC-Acústico, permite gerar modelos de propriedades baseados em $v_{p}$ bastante realísticos. Além desta característica, o emprego da ferramenta Combina Volumes adere detalhes geológicos que permitem aliar modelos conceituais, evolução geológica e propriedades atuais, o que consideramos um avanço na interpretação quantitativa.

Em termos de posicionamento dos refletores, a migração RTM dos dados modelados gerou uma imagem com ótima correlação com a imagem migrada com os dados reais. Tal concordância se dá devido à ótima compatibilidade cinemática entre o dado real e o dado modelado, que fez uso dos insumos gerados pela metodologia SISMEC-Acústico.

\section{Agradecimentos}

Os autores gostariam de agradecer à Neiva Terezinha Zago pela defesa, idealização e disponibilização de recursos humanos e materiais, fundamentais para 0 nascimento e execução do projeto e à Luís Henrique Amaral pelo apoio no desenvolvimento do projeto e disponibilização de recursos humanos; aos colegas Tiago Cabral Luiz, Eduardo Soares Ferreira que contribuem e contribuíram para o avanço do projeto; aos colegas Carlos Cunha, Cláudio Guerra, Márcia Pimentel e Bruno Dias pelas sugestões e discussões promovidas; e aos colegas interpretes Adriana de Oliveira Gangá e Bruno Catto pelas discussões e sugestões sobre o modelo e a geologia associada. Finalmente os autores agradecem à CGG pela cessão e disponibilização de uso das imagens sísmicas.

\section{Referências}

Alves, G.C., Bulcão, A., Soares Filho, D.M., Theodoro, C. Santos, L.A., M.A.G., Guimaraes, 2008, Target oriented illumination analysis using wave equation, SEG Technical Program Expanded Abstracts, 163-167.

Bulcão, A., 2004, Modelagem e migração reversa no tempo empregando operadores elásticos e acústicos, Tese de doutorado, Universidade Federal do Rio de Janeiro.

Carcione, J., 2002, Seismic modeling, Geophysics, v.67, n.4, p. 1304-1325.

Cunha Filho, C.A., 1992, Elastic modeling and migration in Earth models, PhD. Thesis, Stanford University.

Duarte, F.S., 2012, Modelagem acústica no domínio da frequência através do emprego de diferentes esquemas de diferenças finitas, Dissertação de mestrado, Universidade Federal do Rio de Janeiro.

Lira, J. E.M., 2004, Avaliação do Efeito do Acamamento na Deformação do Sinal Modelagem Numérica e 
Calibração com Experimento Físico, Dissertação de mestrado, Universidade Federal do Rio de Janeiro.

Martins, E.O., 2003, Modelagem sísmica em meios complexos, Dissertação de mestrado, Universidade Federal do Rio de Janeiro.

Rosa Filho, J.C., 2002, Modelagem Sísmica de Ondas Elásticas e Migração Reversa no Tempo em Meios Transversalmente Isotrópicos, Dissertação de mestrado, Universidade Federal do Rio de Janeiro.

Santos, L.A., Moraes, A., Silva, A. T., Carneiro, V.F., Carvalho, P. M., Ruthner, M. P., Fraquelli, H. A., 2017, Seismically guided exploration geomechanical model: 15th International Congress of Brazilian Geophysical Society \& EXPOGEF. 

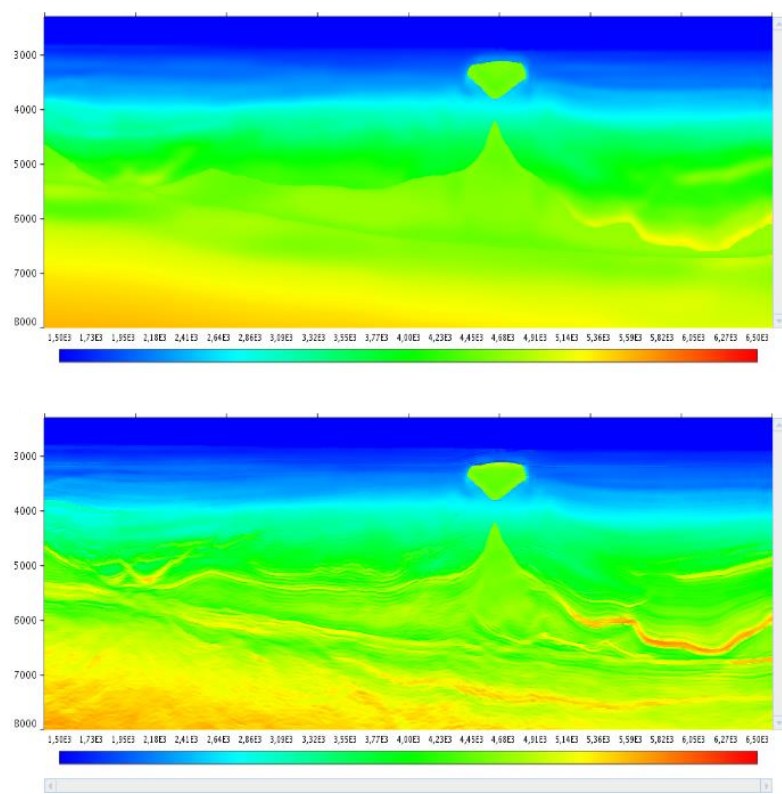

Figura 3. Acima: Velocidade $\mathrm{P}$ do processamento inicial. Abaixo: Velocidade $P$ recalculada após fluxo de inversão acústica.
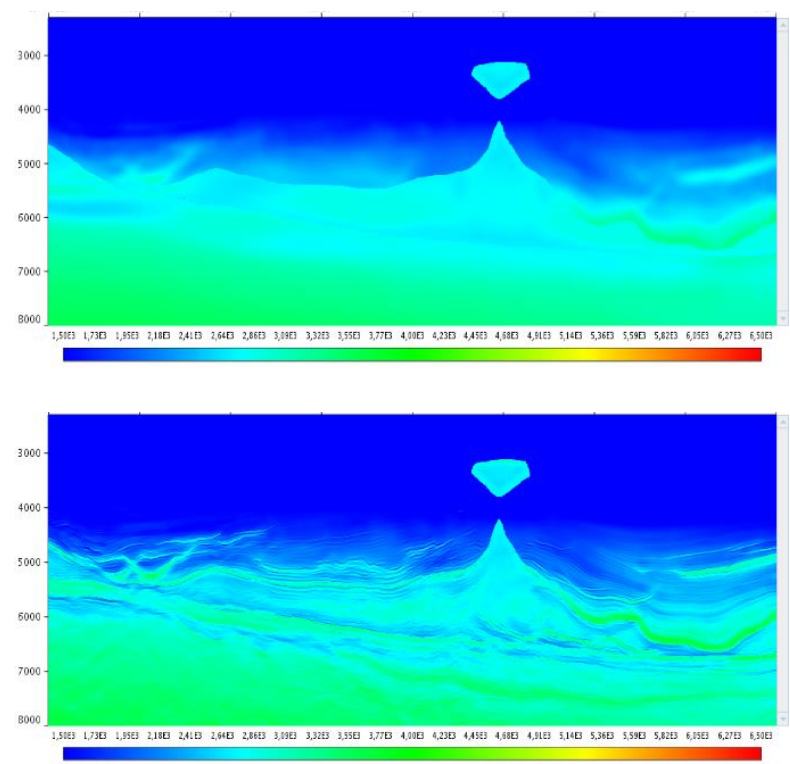

Figura 4. Acima: Velocidade $S$ do processamento inicial. Abaixo: Velocidade $S$ recalculada após fluxo de inversão acústica.
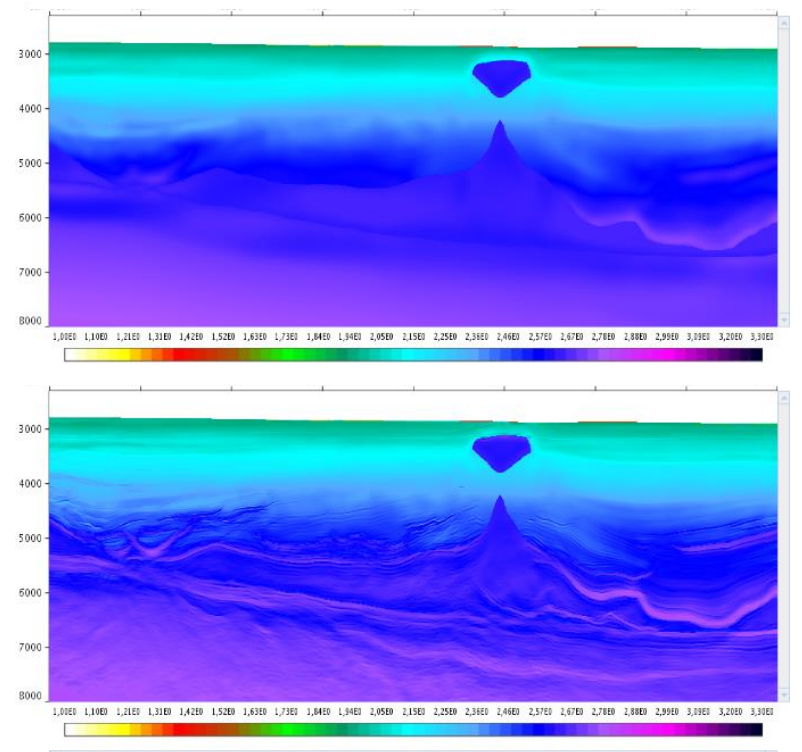

Figura 5. Acima: Densidade do processamento inicial. Abaixo: Densidade recalculada após fluxo de inversão acústica.
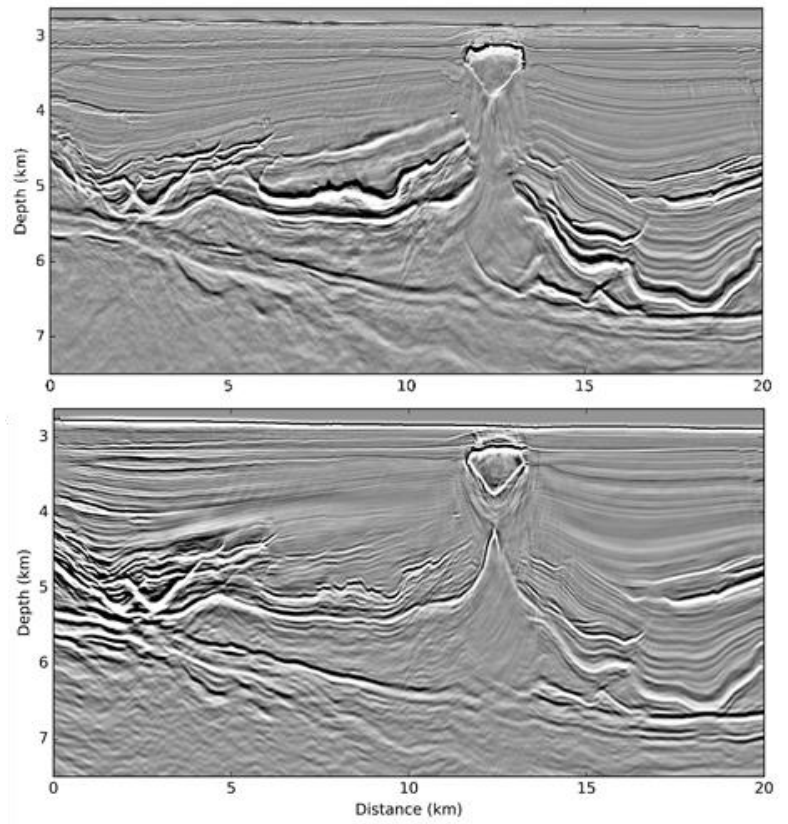

Figura 6. Acima: Resultado da migração RTM (cortesia da CGG) no dado real. Abaixo: Resultado da migração RTM usando o dado elástico isotrópico gerado pelo programa de propagação elástica. 